\title{
Color and animation preferences for a light band eHMI in interactions between automated vehicles and pedestrians
}

\section{Citation for published version (APA):}

Dey, D., Habibovic, A., Pfleging, B., Martens, M. H., \& Terken, J. M. B. (2020). Color and animation preferences for a light band eHMI in interactions between automated vehicles and pedestrians. In CHI 2020 - Proceedings of the $2020 \mathrm{CHI}$ Conference on Human Factors in Computing Systems [198] Association for Computing Machinery, Inc. https://doi.org/10.1145/3313831.3376325

DOI:

10.1145/3313831.3376325

Document status and date:

Published: 21/04/2020

\section{Document Version:}

Publisher's PDF, also known as Version of Record (includes final page, issue and volume numbers)

\section{Please check the document version of this publication:}

- A submitted manuscript is the version of the article upon submission and before peer-review. There can be important differences between the submitted version and the official published version of record. People interested in the research are advised to contact the author for the final version of the publication, or visit the $\mathrm{DOI}$ to the publisher's website.

- The final author version and the galley proof are versions of the publication after peer review.

- The final published version features the final layout of the paper including the volume, issue and page numbers.

Link to publication

\section{General rights}

Copyright and moral rights for the publications made accessible in the public portal are retained by the authors and/or other copyright owners and it is a condition of accessing publications that users recognise and abide by the legal requirements associated with these rights.

- Users may download and print one copy of any publication from the public portal for the purpose of private study or research.

- You may not further distribute the material or use it for any profit-making activity or commercial gain

- You may freely distribute the URL identifying the publication in the public portal.

If the publication is distributed under the terms of Article 25fa of the Dutch Copyright Act, indicated by the "Taverne" license above, please follow below link for the End User Agreement:

www.tue.nl/taverne

Take down policy

If you believe that this document breaches copyright please contact us at:

openaccess@tue.nl

providing details and we will investigate your claim. 


\title{
Color and Animation Preferences for a Light Band eHMI in Interactions Between Automated Vehicles and Pedestrians
}

\author{
Debargha Dey ${ }^{1}$, Azra Habibovic ${ }^{2}$, Bastian Pfleging ${ }^{1,3}$, Marieke Martens ${ }^{1}$, Jacques Terken ${ }^{1}$ \\ ${ }^{1}$ Eindhoven University of Technology, Eindhoven, The Netherlands \\ ${ }^{2}$ Research Institutes of Sweden (RISE), Gothenburg, Sweden \\ ${ }^{3}$ LMU Munich, Munich, Germany \\ d.dey@tue.nl, azra.habibovic@ ri.se, \{b.pfleging,m.h.martens,j.m.b.terken\}@tue.nl
}

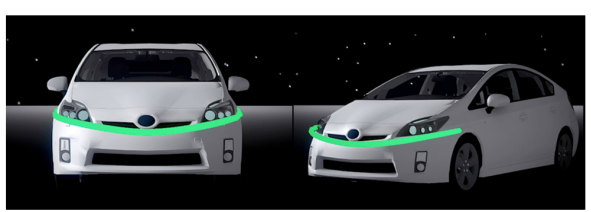

Green

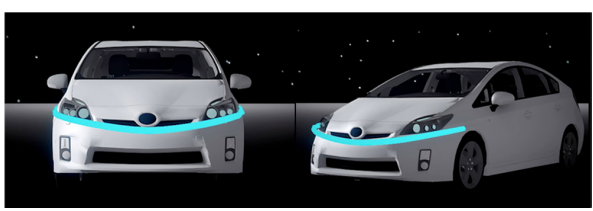

Cyan

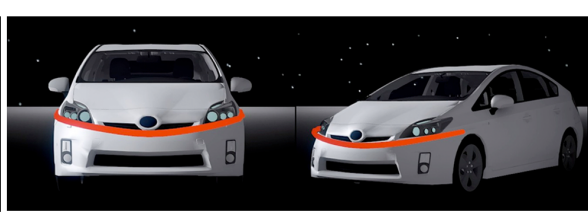

Red

Figure 1. We evaluated user preferences for a light band eHMI with 3 colors (green, cyan, and red), and 5 animation patterns (flashing, pulsing, wiping inwards, wiping outwards, and wiping alternatively inwards as well as outwards).

\section{ABSTRACT}

In this paper, we report user preferences regarding color and animation patterns to support the interaction between $\mathrm{Au}$ tomated Vehicles (AVs) and pedestrians through an external Human-Machine-Interface (eHMI). Existing concepts of eHMI differ - among other things - in their use of colors or animations to express an AV's yielding intention. In the absence of empirical research, there is a knowledge gap regarding which color and animation leads to highest usability and preferences in traffic negotiation situations. We conducted an online survey $(\mathrm{N}=400)$ to investigate the comprehensibility of a light band eHMI with a combination of 5 color and 3 animation patterns for a yielding AV. Results show that cyan is considered a neutral color for communicating a yielding intention. Additionally, a uniformly flashing or pulsing animation is preferred compared to any pattern that animates sideways. These insights can contribute in the future design and standardization of eHMIs.

\section{Author Keywords}

Automated Vehicles; Autonomous Vehicles; Pedestrians; VRU; eHMI; Interface; Color; Animation.

\section{CCS Concepts}

-Human-centered computing $\rightarrow$ User studies; Empirical studies in HCI;

\footnotetext{
Permission to make digital or hard copies of all or part of this work for personal or classroom use is granted without fee provided that copies are not made or distributed for profit or commercial advantage and that copies bear this notice and the full citation on the first page. Copyrights for components of this work owned by others than ACM must be honored. Abstracting with credit is permitted. To copy otherwise, or republish, to post on servers or to redistribute to lists, requires prior specific permission and/or a fee. Request permissions from permissions@acm.org.

CHI '20, April 25-30, 2020, Honolulu, HI, USA.

Copyright is held by the owner/author(s). Publication rights licensed to ACM. ACM ISBN 978-1-4503-6708-0/20/04 _..\$15.00.

http://dx.doi.org/10.1145/3313831.3376325
}

\section{INTRODUCTION}

In current traffic, drivers and other road users communicate in one of two ways: (1) Vehicle-centric cues such as the vehicle's velocity, deceleration, and stopping distances [3, 4, 36, 48], and (2) driver-centric cues such as gestures, eye-contact, and posture $[15,19,20,35,36,39,40,43]$. It has been argued that in the absence of driver-centric communication, automated vehicles (AVs) may need external Human-Machine-Interfaces (eHMI) to communicate safely and effectively with pedestrians in road-crossing situations $[9,26]$. Recent research and development have produced several concepts of eHMI design $[6,8,33,41]$ which vary in their implementation and form factors. Of these, the form factor of a one-dimensional light band $[12,18,21,22,28,42]$ is particularly popular by virtue of being relatively simple and easy to implement within the current design language of a car. Recent studies have shown mixed results regarding the effectiveness of this design when compared to other designs of eHMI $[1,9,14,22,23$, $25,34]$. However, despite the absolute efficacy of this design being questionable, its sheer popularity makes it worthwhile to investigate finer details of its implementation.

Currently, there is no consensus regarding the color or animation pattern that should be used for crucial communication messages within this design. Concepts have showcased light band eHMIs that glow steadily, light up in a pattern, or move continuously to communicate the message that an AV is yielding. Furthermore, this design has been proposed and prototypes implemented in various colors, including white, amber, magenta, green, and cyan. Not only can this lack of standardization lead to ambiguity, but in the absence of empirical research, there is a gap in the knowledge regarding which color and animation pattern yield highest usability and preferences ratings in in traffic negotiation situations. 
We conducted an online crowdsourcing survey to investigate the efficacy of 3 colors and 5 animation patterns on a light band eHMI to convey a yielding intention. We found that red and green have strong, but sometimes contradictory associations of 'stop' and 'go' in relation to either the vehicle or the pedestrian, and may be dangerous in eHMI applications.

\section{Contribution Statement}

The findings from our online survey $(\mathrm{N}=400)$ contribute to the body of knowledge in the design of eHMIs for AVs by identifying the suitability and making recommendations for colors and patterns for use in eHMIs to communicate a yielding message to pedestrians.

\section{Background}

\section{Light Color for eHMls}

Recent research has made several recommendations regarding color choices of light-based eHMIs. Colors for communication from an AV's eHMI should not interfere with colors already implemented or reserved for other purposes in vehicles according to the specifications of SAE J578 Standard [37] and UNECE Regulation R-65 [45]. This prohibits the use of Red, Yellow (Amber), Selective Yellow, Green, Restricted Blue, Signal Blue and White. The task forces from SAE [38] and UNECE [46] on lighting and signaling requirements for automated vehicles show some initial findings regarding the advantages and disadvantages of using certain color spectrums for AV-road user communication [44, 49]. Analyses in different dimensions of light perception such as visibility, discriminability, and uniqueness suggest that a shade of blue-green, or turquoise, or cyan is well-adapted as a color of choice for eHMI to communicate an AV's intention in traffic. However, there is still a knowledge gap regarding the contextual efficacy of the recommended cyan color in an eHMI to communicate with other road users.

Given that cyan has gained favor as a color of choice for eHMI design from a technical point of view, the question arises whether cyan also has any intuitive connotations by nature. Red and green are commonly-used colors in traffic lights, and over time have come to be associated with the messages "stop" and "go". Although currently established laws and recent research already recommend that red and green lights may not be used on the front of a car, it is interesting to investigate what the innate associations of intended traffic behavior are when these lights are mounted on a moving vehicle as opposed to a stationary traffic light. Recently, Petzoldt et al. showed in a study that a car illuminating a green light in its front to indicate braking behavior had a positive effect on pedestrians [34]. Regardless of legal implications, a common point of confusion from a human factors perspective when red or green lights are mounted on a moving vehicle is the frame of reference from whom these lights are meant to communicate. Would a red light indicate that the vehicle itself is stopping, or is it advising other road users to stop? Conversely, would a green light indicate the car's intention to let other road users go, or its own intention of going? It is thus interesting to investigate what the preconceived associations with red and green colored lights on a moving vehicle are, and how cyan fits among them.

\section{Animation patterns in eHMI}

Besides color, the animation pattern in which the light band illuminates may have certain connotations and inherent meanings in the traffic context. Blinking and flashing patterns are already commonly used in cars in hazard lights or to indicate turning intention. Additionally, flashing the headlights of a car is a common explicit communication tool used by drivers to negotiate with pedestrians and other road users. Furthermore, in the recent past, some vehicles have adopted the use of laterally sweeping animation patterns to indicate turning intention (sequential turn signals) [30,47]. Emergency vehicles also typically use various forms or rotating or flashing lights to warn other traffic of their presence. Given this existing context, it is important to investigate whether - in scenarios involving future $\mathrm{AVs}$ that may also attempt to communicate with pedestrian using some pattern of vehicle lighting - pedestrians have certain expectations or intuitive associations regarding the meaning behind certain animations.

Previous research has explored different ways an eHMI can activate in order to communicate different states in an AV's operating cycle [29]. Hensch et al. [24, 25] has shown that a light bar with two segments of lights that animate sideways has potential as an effective communicator of yielding intention to VRUs. In contrast, another recent study [14] shows that steady or flashing lights in eHMI yield better results than sweeping lights. Among these contradicting results, we aim to extend the body of work by exploring the user preferences of color and animation of a light-band eHMI.

The one-dimensional form factor of a light band eHMI does not leave a lot of room for different animation patterns to be implemented in it. However, the flashing and sweeping/wiping patterns that are already used in current traffic can be adapted to this form factor and extrapolated to a few different ways of animation through which a message may be communicated. Thus, we selected 5 different patterns of animation based on the basic flash and sweep animations to evaluate their intuitiveness. While an eHMI may be needed to communicate several messages during an AVs active driving cycle (such as cruising in automated mode, yielding, starting to drive, etc.), we chose to focus on the yielding message from the AV as this is when crucial $\mathrm{AV}$-pedestrian negotiations will take place. However, a yielding vehicle may negotiate its intention with pedestrians in one of two ways: (1) by announcing its own intention ("I am yielding"), or (2) by instructing or advising an action to pedestrians ("Please cross"). While the research community is fairly united in the recommendation that an AV should not issue an advice or instruction to others in traffic [2, 27], it is not known whether there are subtle differences between how an advice or a simple intention announcement is perceived by pedestrians in traffic. Thus, although the message for both ways of communication (intention communication/ instruction) are the same in principle, it is further interesting to investigate if a color/animation combination has innate associations with one or the other.

\section{Research Question and Hypotheses}

Until now, no research has systematically looked into the effectiveness of colors and/or animation patterns in a one- 
dimensional light-bar eHMI from a user-centered design perspective. In this study, we aim to address this research gap with the following research question:

Which color and/or animation pattern on a light-band eHMI is the most favorable according to users for a yielding message from an $A V$ towards a pedestrian?

We conducted an online survey to study the user preferences of 3 colors and 5 animation patterns across 2 communication messages (intention announcement/ instruction). Our hypotheses were as follows:

- H1: Red will not be intuitive to convey a yielding message from an AV's eHMI. Green will be intuitive to communicate an AV's yielding intention. Cyan will fall somewhere in the middle.

Given the current association of red and green lights with "stop" and "go" respectively in traffic lights, this may extend to moving vehicles as well. Cyan has no current usage in traffic and in the lack of an existing association, its intuitiveness for a yielding message will likely be neutral.

- H2: Wiping or sweeping animation patterns will be more intuitive for an yielding message than a uniformly animating pattern.

Laterally sweeping animation patterns may have an abstract similarity with gestures from a driver such as a sweeping hand motion used to indicate a driver's willingness to let a pedestrian go first. Thus, applying Wickens's model of display design [50], a sweeping animation may map strongly to users' mental models and score higher in intuitiveness.

\section{METHOD}

\section{Design}

The experiment was conducted in a mixed design with three independent variables (two within-subjects, and one betweensubjects), and one dependent variable. The two within-subjects independent variables were Color with the three levels green, red, and cyan as shown in Figure 1, and Animation with five different levels (Flash, Pulse, InSweep, OutSweep, DualSweep) as shown in Figure 2. The between-subjects independent variable was Message (Intention communication, Instruction). The dependent variable was the user's score of the intuitiveness of a shown eHMI for a yielding message for a car on a Likert scale of 1 to 5 , as well as their subjective opinions on what drives this intuitiveness.

\section{Participants}

The experiment sample consisted of 400 participants with 200 in each condition of the between-subjects factor (Intention communication vs. Instruction). We recruited the participants using Amazon Mechanical Turk (MTurk) [7]. In order to ensure the quality of the responses, only MTurk Master Workers were recruited. "A Master Worker is a top Worker of the MTurk marketplace that has been granted the Mechanical Turk Masters Qualification. These Workers have consistently demonstrated a high degree of success in performing a wide range of HITs across a large number of Requesters"1. Recent

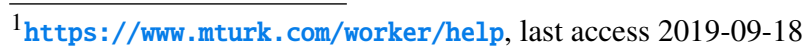

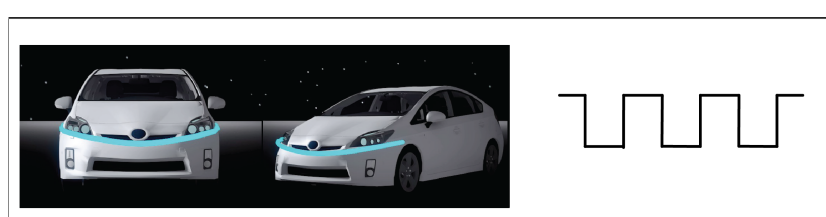

Flash: The light band uniformly and alternately glows with full brightness and then turns off
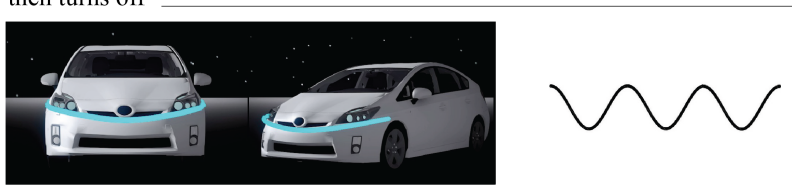

Pulse: The light band uniformly glows and dims in a sinusouidal pattern of increasing and decreasing brightness

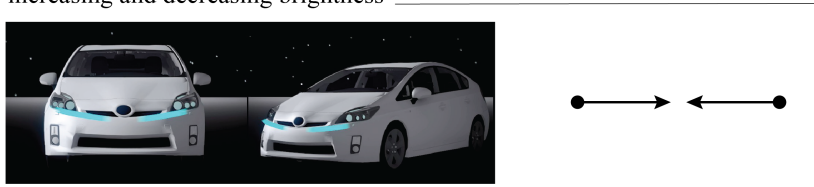

InSweep: The light band animates in an inward sweeping pattern of two light segments (edges of the bumper to the middle)

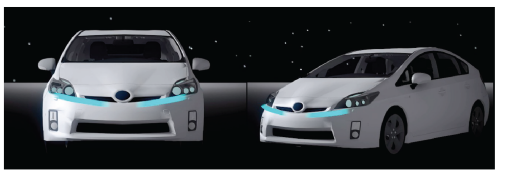

OutSweep: The light band animates in an outward sweeping pattern of two light segments (middle to the edges of the bumper)

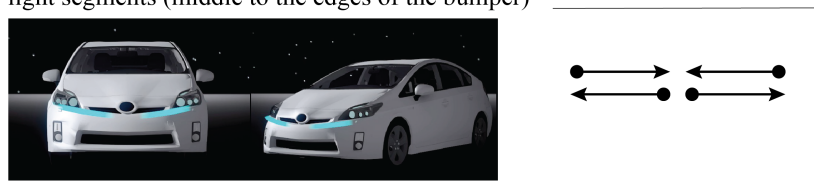

DualSweep: The light band animates in an alternately inward and outward sweeping pattern of two light segments

Figure 2. The 5 animation patterns studied in this experiment represented in the cyan color.

research shows that the MTurk worker demographic is highly skewed towards US and Indian citizens [13], which typically leads most tasks to be picked up by workers from these two countries. Given that traffic and automobile cultures are very different in the US and India; in order to draw meaningful conclusions and ensure homogeneity and ecological validity of the responses, we recruited participants only from the US. In order to ensure that the participants had an acceptable understanding of the context and task, we conducted a manipulation check at the end of the survey. Furthermore, the data was also scrutinized for any obvious discrepancies or inconsistencies (as discussed in the Results section below). This resulted in omitting responses from 17 participants from the Intention communication condition, and 8 participants from the Instruction condition. The distribution of the participants with valid responses for each condition is shown in Table 1.

\section{Experimental Setup}

As a medium for the light band eHMI, we used the model of a Toyota Prius, and we modeled the eHMI on the bumper of the vehicle in Unity $3 \mathrm{D}^{2}$. We placed the vehicle in a featureless background devoid of any context - there were no road, scenery, or speed information. This was done to ensure that

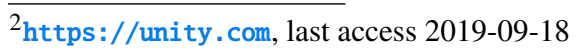




\begin{tabular}{||l|l|l||}
\hline Age group & $\begin{array}{l}\text { Intention } \\
\text { Communication } \\
(53.2 \% \text { male })\end{array}$ & $\begin{array}{l}\text { Instruction } \\
(44 \% \text { male })\end{array}$ \\
\hline \hline $18-24$ & 4 & 3 \\
$25-34$ & 60 & 69 \\
$35-44$ & 67 & 66 \\
$45-54$ & 28 & 31 \\
$55-64$ & 16 & 17 \\
65 and above & 8 & 6 \\
\hline \hline Total & 183 & 192 \\
\hline
\end{tabular}

Table 1. participant demographics

the intuitiveness of the eHMI's yielding message can be scored entirely on its color and pattern, and would not be influenced by other factors. A video of each eHMI in action (animating in a certain pattern and color combination) was displayed from two angles: front (head on), and front three-quarters as shown in Figure 3. We chose the front view so that an unobstructed view of the eHMI can be observed by the participants, while the front three-quarters view helps the participants to perceive the car like a pedestrian would observe it while standing at the edge of a sidewalk.

We presented the experiment as a questionnaire on the online survey platform SurveyGizmo ${ }^{3}$ and then deployed it through MTurk.

With the 3 colors and 5 animation patterns tested, each participant experienced 15 videos during the experiment. In order to provide the participants with some reference for comparison, we presented the stimuli of the eHMI either in groups of colors or animation patterns. Each page of the questionnaire was dedicated to either one color or one animation pattern. Thus the entire questionnaire consisted of 8 pages -3 pages for the different colors, and 5 pages for the different animations. For each of the 3 colors, we showed the 5 animation patterns next to each other on the same page. Conversely, for each of the 5 animation patterns, we showed the 3 colors next to each other on the same page. This led each color/animation combination of an eHMI to be graded twice: once from the perspective of its color, and once from the perspective of its animation pattern. This also allowed us to see whether the perspective from which an eHMI was scored for its intuitiveness made a difference.

The survey introduced the concept of Automated Vehicles in short, adapted from [11]. It then explained that in the absence of a driver, AVs may communicate with pedestrians with light on the front of the vehicles. Next, we asked the participants to regard the light from a pedestrian's point of view who wants to cross the road, and requested them to rate the eHMIs regarding their intuitiveness to a yielding message from a car. We emphasized that there is no 'incorrect' answer, and encouraged the participants to respond spontaneously without overthinking.

Each page of the questionnaire asked the participants to consider the following videos of eHMI and rate their intuitiveness.

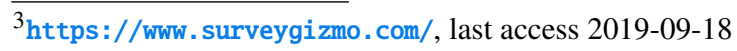

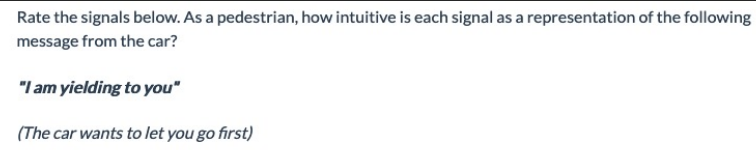

(The car wants to let you go first)

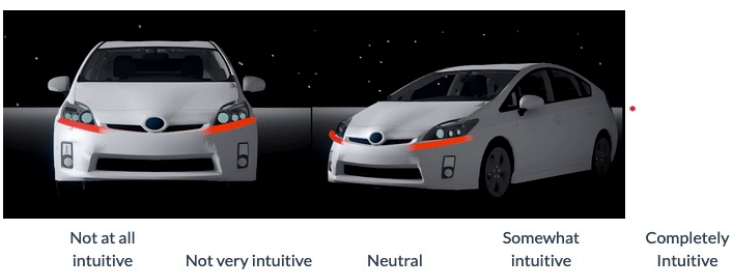

$\begin{array}{ccccc}\text { intuitive } & \text { Not very intuitive } & \text { Neutral } & \text { intuitive } & \text { Intuitive } \\ 0 & 0 & 0 & 0 & 0\end{array}$
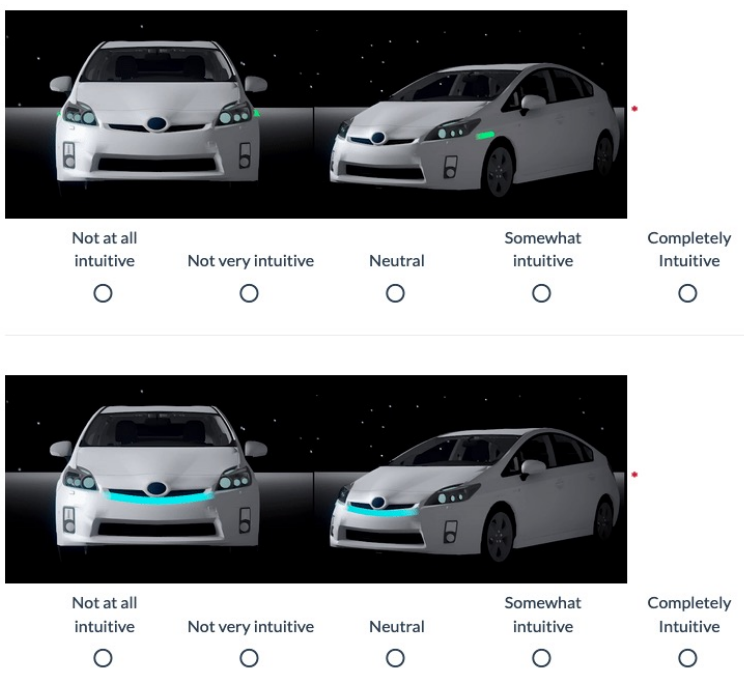

Back Next

Figure 3. A screenshot of the layout of the questionnaire. This page asks participants to rate the intuitiveness of the 3 colors for the same animation pattern (in this case, DualSweep) in the Intention announcement condition.

The exact phrase used to elicit the responses from the participants was as follows:

Rate the signals below. As a pedestrian, how intuitive is each signal as a representation of the following message from the car?

$<$ MESSAGE $>$

(The car wants to let you go first)

The $<$ MESSAGE $>$ was manipulated between the two conditions as follows:

- Intention announcement: "I am yielding to you"

- Instruction: "Please cross the road"

This was followed by the videos of the eHMIs being tested in the group, each of which was supplemented with a 5-point Likert scale for participants to indicate their intuitiveness. An example of the layout of the question is shown in Figure 3.

We randomized the order of the pages and the order of each video within each page to counterbalance any learning effects. 
After the scoring of the eHMIs' intuitiveness was complete, we requested the participants to fill out their subjective opinions about what made an eHMI intuitive in an open, free-form text box, which would serve as qualitative data for our insights. The survey closed with some demographic questions.

One difficulty with using crowdsourcing to gather scientific data for research is the question whether the instructions were successfully conveyed to the participants. Long and dense introductions may lead participants to skip reading parts of the instruction, which might lead them to miss important information. Thus, we took care that the instructions were as short and succinct as possible. However, it was important to make sure that the conciseness of the instructions did not interfere with a thorough understanding of the question and the task. Thus, at the end of the survey, we asked several questions as a manipulation check. We verified whether the participants understood two elements: (1) that the responses were to be given from the point of view of a pedestrian (as opposed to cyclists or other drivers), and (2) that the message they were evaluating the eHMI towards was that the car was yielding/letting them cross first (as opposed to the car cruising in automated mode, or starting to drive from rest). In order to control for participants' perception of color without having to ask for medical information, we added another manipulation check at the end of the study where we asked the participants to report the colors of the eHMI they observed in the study.

A pilot study conducted with 5 people before deployment showed that the survey took between 8 and 12 minutes to complete. Accordingly, we compensated each worker with 2.00 USD for their participation. Since we conducted the experiment as a between subjects study, we deployed the Intention communication condition of the study first and allowed it to run until the necessary number of responses (200) was reached. After receiving these 200 responses, we deployed the second condition (Instruction) by excluding any Worker who had already participated in the previous condition.

\section{Limitations}

The major limitations of this study are embedded in the study approach itself. We selected MTurk as a cost-efficient methodology enabling data collection from a rather large and varied participant sample; however, using online crowdsourcing is not without challenge. As highlighted before, to ensure the quality of the responses, we only recruited MTurk Master Workers. They were also subjected to a manipulation check to ensure basic understanding of the task and context, as well as color perception. However, this does not warrant that the survey was, for instance, answered with full attention or with great honesty. The fact that the participants got paid for participating amplifies the risk for such issues. However, we think that this risk is not much higher compared to distribution an online survey through other channels. Furthermore, the videos used in the survey were on purpose decontextualized (e.g., they did not display any traffic situation nor environment) which might have made it more difficult for the participants to envision the function of the eHMI, especially if considering that AVs are a new phenomenon and that few people have experience of interacting with them. However, using decon- textualized material enabled us to study the effect of color and pattern in isolation from other potentially confounding factors. Based on these limitations, however, we recognize a need for conducting more contextualized studies as well as assessing the capability of MTurk to replicate results from corresponding studies where participants are selected in a more controlled manner.

\section{RESULTS}

\section{Manipulation Check and Data Analysis}

The responses to the manipulation check questions showed that most participants understood the context and the task of the question correctly. The participants who failed to answer these questions correctly were omitted from the data analysis. The 5-point intuitiveness scale responses per participant were aggregated, and checked for variances within individuals across the different eHMI colors and animations. Some responses showed little to no variation among the perception of different color/animation conditions - i.e., some participants felt that irrespective of the eHMI property, every eHMI they encountered were equally intuitive or equally unintuitive. Thus, the Standard Deviation of the responses for each participant was calculated, and all participants for whom response $\mathrm{SD}<0.5$ were also omitted from the analysis. Essentially, for these participants, eHMI colors or animation patterns did not make any difference. We analyzed the rest of the data from participants for whom the eHMI color or pattern had a meaningful difference $S D>0.5$. This led to a slightly reduced participant pool for the analysis form the original 200 per condition ( $\mathrm{N}=183$ for the Intention communication condition, and $\mathrm{N}=192$ for the Instruction condition).

The rest of the data were subjected to analysis using parametric tests. Although opinion is divided regarding whether Likert scale data is suitable for parametric tests, research suggests that for data from Likert scales with 5 or more points and with a large number of participants ( $>30)$, application of parametric tests lead to negligible Type I or Type II errors [10, $31,32]$. The analysis was done in 3 steps. In the first two steps, the results from the two studies corresponding to each of the two between-subjects factors of the Message (Intention communication and Instruction) were analyzed separately using repeated-measures ANOVA. In the third and final step, a mixed ANOVA was conducted to analyze the impact of Message since the study design across the Message was identical, and the overall effects were used to inform overarching insights. While this multi-step analysis raises a potential danger of multiple-comparison issues, the results of the mixed ANOVA are used to draw a holistic picture from the two independent studies. We report only the statistically significant effects and interactions for the sake of brevity.

\section{Results of Intention Communication}

We used a repeated-measures ANOVA to test the effects of the colors and animation patterns of the eHMI for the Intention communication message. Since we collected the responses to an eHMI's intuitiveness twice (different colors for the same animation, and different animations for the same color), we considered this as another independent variable 'Measure' to 


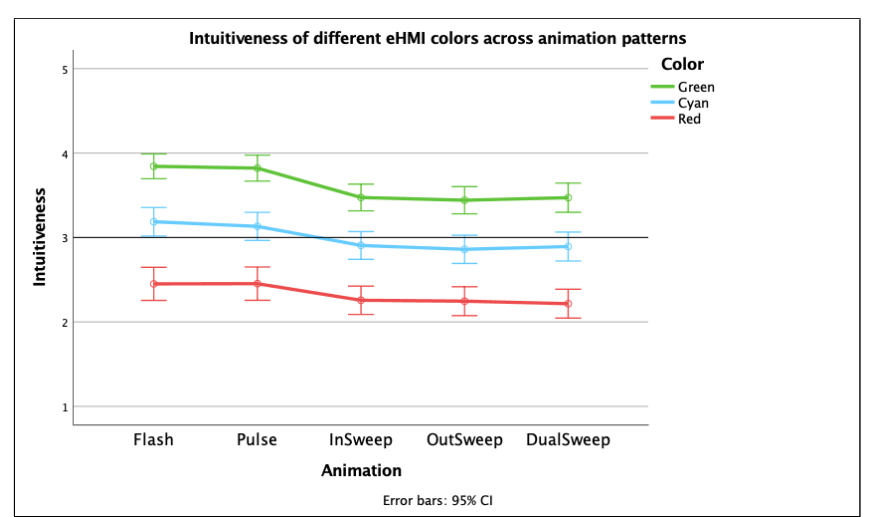

Figure 4. Intuitiveness of different eHMI colors and animation patterns for the Intention Communication condition. Intuitiveness is scored from 1 (Not intuitive at all) to 5 (Completely intuitive), with 3 being Neutral.

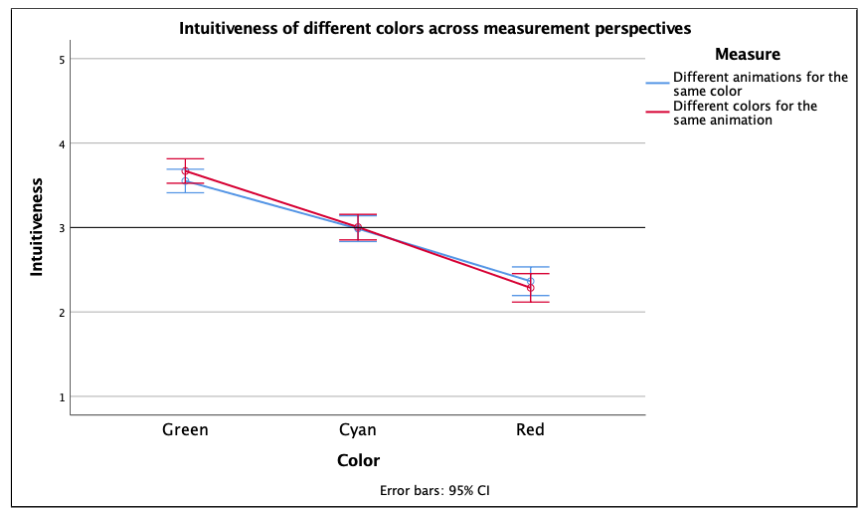

Figure 5. The effect of measurement perspectives on intuitiveness of eHMI color in the Intention announcement condition. Intuitiveness is scored from 1 (Not intuitive at all) to 5 (Completely intuitive), with 3 being Neutral.

investigate whether the perspective of evaluation of the eHMI had any effect on the eHMI's intuitiveness score. Thus, there were 3 independent variables (Color, Animation, and Measure) and one dependent variable Intuitiveness. Mauchly's test indicated that the assumption of sphericity had been violated for both eHMI Color $\left(\chi^{2}(2)=73.82, \mathrm{p}<0.001\right)$, and Animation $\left(\chi^{2}(9)=484.07, \mathrm{p}<0.001\right)$. Therefore, multivariate tests are reported as they do not assume sphericity [16, 17] $\left(\varepsilon_{\text {color }}=0.751\right.$, and $\left.\varepsilon_{\text {animation }}=0.405\right)$. The results showed a significant main effect of the eHMI Color on intuitiveness $\left(\mathrm{F}(2,183)=62.34, \mathrm{p}<.001, \eta_{p}^{2}=.41\right)$. The intuitiveness of the eHMI was also significantly affected by the Animation $\left(\mathrm{F}(4,181)=6.57, \mathrm{p}<.001, \eta_{p}^{2}=.13\right)$. The interaction Color * Animation was not significant, but the overview of the interaction is shown in Figure 4. The variable Measure (whether the intuitiveness of the eHMI was scored from the perspective of the color or animation) had a significant interaction with Color $\left(\mathrm{F}(2,183)=7.44, \mathrm{p}=.001, \eta_{p}^{2}=.08\right)$, and an effect size of 0.08 shows a medium effect. This interaction is shown in Figure 5. No other factors or interactions were statistically significant.

\section{Results of Instruction}

Similarly as the Intention communication condition, a repeated-measures ANOVA was used to test the effects of

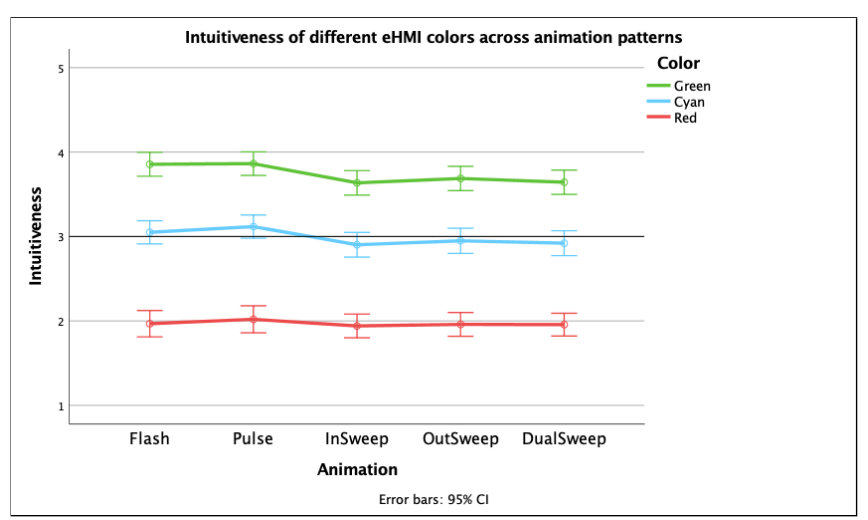

Figure 6. Intuitiveness of different eHMI colors and animation patterns for the Instruction condition. Intuitiveness is scored from 1 (Not intuitive at all) to 5 (Completely intuitive), with 3 being Neutral.

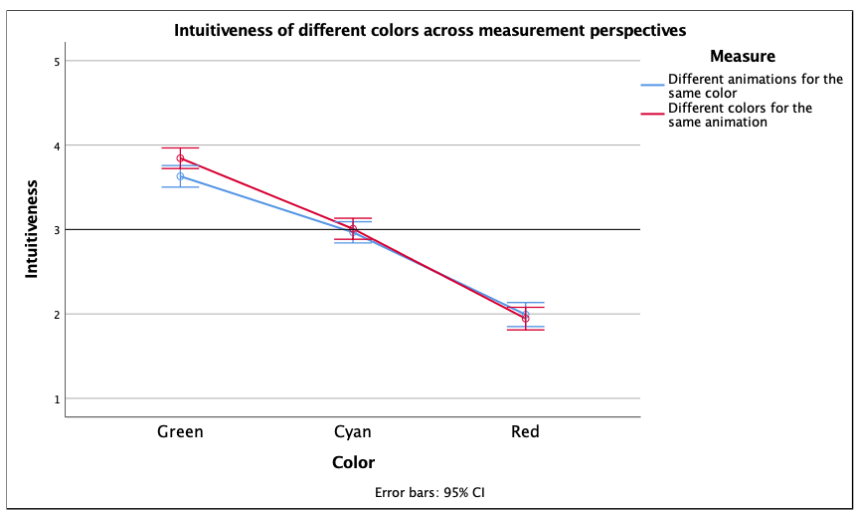

Figure 7. The effect of measurement perspectives on intuitiveness of eHMI color in the Instruction condition. Intuitiveness is scored from 1 (Not intuitive at all) to 5 (Completely intuitive), with 3 being Neutral.

the Color and Animation patterns of the eHMI for the Instruction message. Once again, there were 3 independent variables (Color, Animation, and Measure), and one dependent variable Intuitiveness. Mauchly's test for sphericity had also been violated in this condition for both eHMI Color $\left(\chi^{2}(2)=42.21, \mathrm{p}<\right.$ 0.001 , and Animation $\left(\chi^{2}(9)=237.83, \mathrm{p}<0.001\right)$. Therefore, multivariate tests are reported as they do not assume sphericity $\left(\varepsilon_{\text {color }}=0.835\right.$, and $\left.\varepsilon_{\text {animation }}=0.568\right)$. The results showed a significant main effect of the eHMI Color on intuitiveness $\left(\mathrm{F}(2,192)=153.01, \mathrm{p}<.001, \eta_{p}^{2}=.61\right)$, demonstrating a very large effect. The intuitiveness of the eHMI was also significantly affected by the Animation $(\mathrm{F}(4,190)=2.98, \mathrm{p}=0.02$, $\left.\eta_{p}^{2}=.06\right)$, but the partial $\eta^{2}$ shows only a medium effect in this condition. The interaction Color * Animation was again not significant, but the overview of the intuitiveness across different colors and animations is shown in Figure 6. Measure (whether the intuitiveness of the eHMI was scored from the perspective of the color or animation) once again had a significant interaction with Color $(\mathrm{F}(2,192)=13.28, \mathrm{p}<.001$, $\left.\eta_{p}^{2}=.12\right)$, and an effect size of 0.12 shows a medium to high effect. This interaction is shown in Figure 7. No other factors or interactions were statistically significant.

\section{Comparing Intention Communication and Instruction}

A mixed ANOVA was used to test the effects of the colors and animation patterns of the eHMI with respect to the between- 


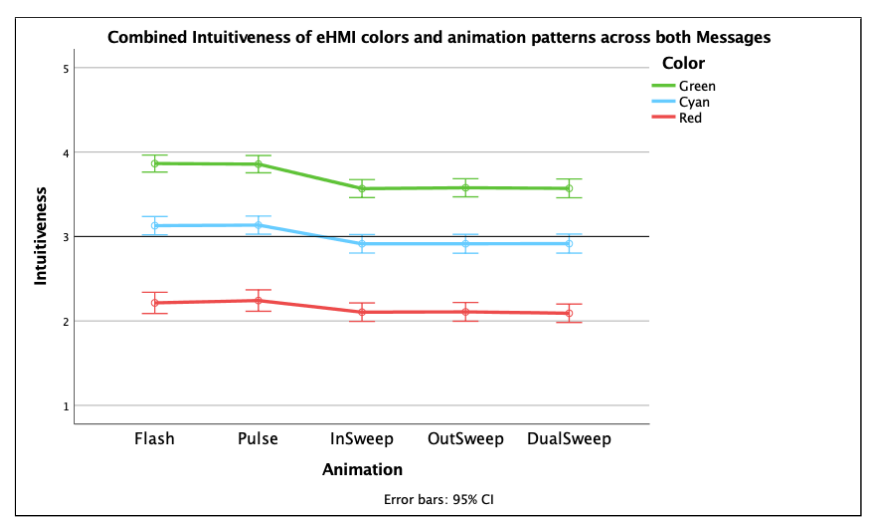

Figure 8. Overall intuitiveness of different eHMI colors and animation patterns across both conditions. Intuitiveness is scored from 1 (Not intuitive at all) to 5 (Completely intuitive), with 3 being Neutral.

subjects conditions of Intention communication and Instruction messages. This led to an analysis with 4 independent variables: 3 within-subjects (Color, Animation, and Measure), and one between-subjects (Message). The dependent variable was once again Intuitiveness.

Mauchly's test once again showed that the assumption of sphericity had been violated for both eHMI Color $\left(\chi^{2}(2)=\right.$ 108.28, $\mathrm{p}<0.001)$ and Animation $\left(\chi^{2}(9)=659.34, \mathrm{p}<0.001\right)$. Therefore, multivariate tests are reported $\left(\varepsilon_{\text {color }}=0.798\right.$, and $\left.\varepsilon_{\text {animation }}=0.479\right)$. Across the two different Messages, the results showed a significant main effect of the eHMI Color on intuitiveness $\left(\mathrm{F}(2,372)=190.23, \mathrm{p}<.001, \eta_{p}^{2}=.51\right)$. The intuitiveness of the eHMI was also significantly affected by the Animation $\left(\mathrm{F}(4,370)=8.21, \mathrm{p}<.001, \eta_{p}^{2}=.08\right)$. The effect sizes of Color was extremely large, while that of Animation was medium, and showed that both these factors had a significant impact in how participants scored the intuitiveness of an eHMI for an yielding message from an AV. Overall, the interaction Color * Animation was significant but had a low effect size $\left(\mathrm{F}(8,366)=1.99, \mathrm{p}=0.047, \eta_{p}^{2}=.04\right)$. The combined overview of the intuitiveness of Color and Animation across the two Messages is shown in Figure 8.

The main effect of the between-subjects condition variable Message (Intention communication/ Instruction) was not significant $\left(\mathrm{F}(1,373)=1.54, \mathrm{p}=0.21, \eta_{p}^{2}=.004\right)$. However, the interaction Color * Message was statistically significant $\left(\mathrm{F}(2,372)=4.89, \mathrm{p}=.008, \eta_{p}^{2}=.03\right)$, and an effect size of 0.03 shows a small to medium effect. This interaction is shown in Figure 9. Post-hoc tests (pairwise comparison of the main effects of the different Colors with Bonferroni confidence interval adjustment) for both the Intention communication and Instruction messages showed that the estimated marginal means for Green, Cyan, and Red were all different from each other in a statistically significant way.

The interaction between Message and Animation was not statistically significant. This interaction is shown in Figure 10 for a comparative overview. Post-hoc tests (pairwise comparison of the main effects of the different Animations with Bonferroni confidence interval adjustment) showed some difference in the effect of the animation patterns on eHMI intuitiveness between the conditions. For Intention communication, while

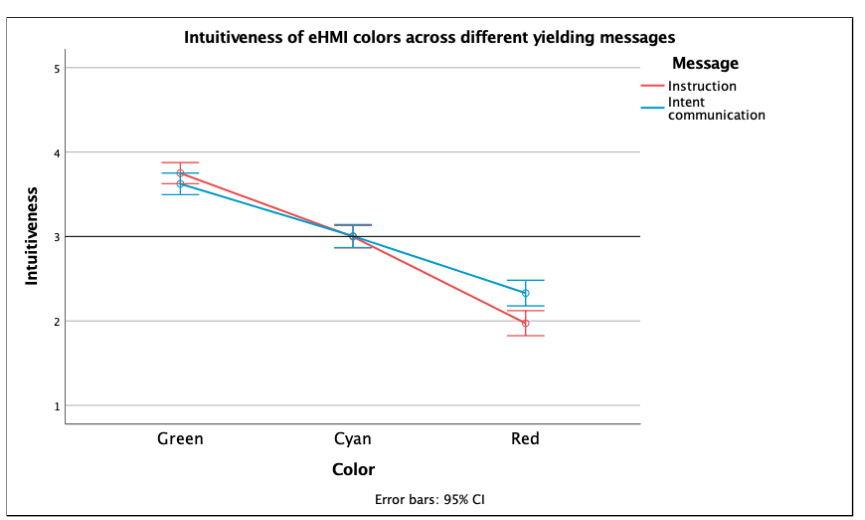

Figure 9. The effect of the Message Intent communication/Instruction on intuitiveness of eHMI color. Intuitiveness is scored from 1 (Not intuitive at all) to 5 (Completely intuitive), with 3 being Neutral.

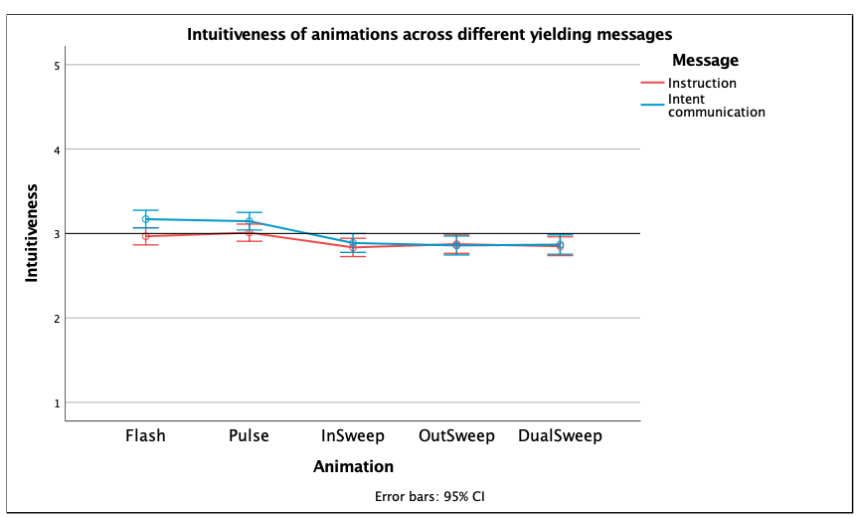

Figure 10. The effect of the Message Intent communication/Instruction on intuitiveness of eHMI animation. Intuitiveness is scored from 1 (Not intuitive at all) to 5 (Completely intuitive), with 3 being Neutral.

each uniform animation (Flash and Pulse) resulted in statistically significantly higher intuitiveness than any sweeping animation (InSweep, OutSweep, or DualSweep), each animation within their own group did not have a significant difference. In contrast, for Instruction, only the Pulse animation scored significantly higher in intuitiveness than InSweep and DualSweep. There were no other significant differences between the animations. However, when the data is combined and analyzed across the two messages, the overall differences suggest that just as in the Intention communication condition, uniform patterns (Flash and Pulse) score higher than sweeping animations (InSweep, OutSweep, DualSweep), although there are no significant differences between themselves.

All other factors or interactions were not statistically significant, and are not reported.

\section{Qualitative Data}

Apart from the quantitative data, qualitative feedback allows us to gain insights from the subjective reasoning of the participants. Responses to the open ended question that asked participants for their reflection on what made a certain eHMI intuitive were subjected to thematic analysis. The insights from this is discussed here, and are furnished with a few relevant participant quotes. In explaining their thought processes, participants often used various words for a blue-green color 
such as 'teal', 'turquoise', or simply 'blue' to refer to the cyan color. For the sake of data integrity, the quotations are furnished unchanged. Remarks made by participants in the Intention communication condition are reported under the prefix 'I', whereas those by participants in the Instruction (Advice) condition are reported under the prefix ' $\mathrm{A}$ '.

\section{Green indicates 'Go'; Red indicates 'Stop'}

An overwhelming majority of the participants commented that the colors green and red led them to make instinctive associations with 'go' and 'stop' respectively as commonly used in traffic lights. Some relevant quotes include:

(I7) "For anything red, it was less likely that I would think that it was intuitive. Red usually signals stop, danger, or do not proceed. So, the red would make me think that the car would not stop or is a danger to me if I were to cross the street. Green is more intuitive because it means 'Go' or something is more safe to proceed."

(I152) "I think that the red is not very intuitive because to me, red automatically means 'stop'. The green that goes all the way across the car's bumper is the most noticeable and the right color for the car to tell a pedestrian to 'go'."

(A9) "I think we are programmed to know that green means go and red means stop. I think that can also be extended to assume that green is a positive and red is a negative. I was more intuitively resigned to choose green to mean to cross the road, whereas I assumed red meant there was something wrong."

(A56) "All my life red has meant stop and green has meant go. If it's safe to cross in front of this car, I would assume any green color would be a sign that things are safe, and red would mean 'don't cross, it's not safe'. Kind of like stop lights."

\section{Sometimes, red and green can lead to confusion}

While most participants embraced the associations of 'go' with green and 'stop' with red, some participants remarked that for commonly used traffic colors applied to a moving vehicle, they would not know whether the notion of stopping or going would apply to the vehicle or to them as a pedestrian. Among the participants who noted their confusion, there were individual differences - for some, only red or only green caused the confusion. Some relevant quotes include:

(I8) "When I saw green, I figured that was a signal that it was safe to walk in front of the car. Red I was confused on. I can understand red meaning it will stop, but it could also be warning me not to cross in front of the car."

(I138) "If I saw the red, I would think the car was stopping which would make me think it was ok to walk. If I saw the green, I would probably think that the car was going to keep driving so I am not so clear on that one. The only issue with me was typically, if you see a red light it means 'stop' and green means 'go'. So I took the perspective of the car telling me what it was doing rather than the car telling me what to do. I figured red meant the car was stopping, green meant the car was in motion."

(A17) "As a pedestrian, it would be hard to figure out if the lights meant what the car was doing, or what the car wanted me to do. The green and red would mean very different things."

(A125) "Regarding color and its comparison to current traffic lights, red is confusing to me. I instinctively think 'stop', but don't intuitively know if I should stop or if the car will stop. A blinking or other pattern in red leads to the same confusion. While a green color could potentially mean either the car will go, or I can go, I intuitively think that a green color is giving me permission to cross the road."

\section{Cyan is considered neutral, but may also be confusing}

Most participants showed some form of a strong bias towards red and green as colors - either from the perspective of the pedestrian or the car. However, cyan did not have a specific preference. Some participants noted that cyan can work to show yielding intention as a neutral color. Others remarked that in the absence of cyan in current traffic, they would not know what significance to attach to it, and this would lead to confusion:

(I124) "I rated the colors from negative (red) to neutral (blue) to green (inviting)."

(I159) "The highest priority for me was the colors. Green indicated go (for the pedestrian) while red indicated stop. Teal was unclear."

(A10) "I liked the blue because I don't already associate it with stop or go. The red could either be that the car is stopped or that I should stop, and green makes it seem like either I can go or the car is going to go forward."

(A107) "Red usually means stop, while green usually means go; so by those standards if a car is blinking red, I would assume it's telling me to stop, while green would make me think to cross. Blue confuses me but is closer to green so it is somewhat understandable."

\section{Color overrides animation patterns}

Most participants noted that the color of the eHMI made a more significant conscious impact in their decision-making compared to the animation patterns. This is corroborated by the following quotes:

(I17) "The patterns mattered less as the survey progressed. The colors stood out to me as the intuitive part."

(I88) "I don't know that any of the patterns are really intuitive except maybe just blinking to grab attention. I would default to the color over the pattern as a signal if I was walking and assume green means for me to go and red means for me to stop."

(A69) "The patterns were not important in my decision, only the colors because I would immediately see the lights and not think about directions they were moving or how long they were lit."

(A131) "The color was the biggest factor... What the lights were doing played a part but not as big a one."

Uniform patterns make more sense over sweeping ones Among participants for whom the animation patterns had an effect, several commented that uniformly changing patterns 
(such as Flash or Pulse) were preferable over the moving or sweeping patterns:

(I52) "I felt like anything blinking was sending the message that "I am waiting for you". Blinking green made the most sense to me. The sliding lines didn't communicate anything to me really because I wasn't sure how they were meant to be interpreted."

(I82) ": I think the solid lights flickering on and off in a solid band of color across the front of the car were most effective as they showed the car was 'waiting'. Having the lights move from the center toward the sides of the car was next - It seemed to me the car was telling me to go ahead and move around the car. However, I could also see where this could [be] confused with a turn signal light, and maybe the car is not waiting to yield at all, but just waiting for its turn (against other vehicles) to go. The option where the lights moved from the sides toward the front of the car seemed least intuitive to me. It seemed like the car was telling me to go to the front of the car, not walk along the crosswalk."

(A73) "The segmented or crossing lines were slightly less intuitive and made it seem like the car was doing something, but I wouldn't be sure it was safe for me to cross or that I was supposed to go first."

(A129) "The solid lights that strobed off and on seemed to indicate a signal, whereas the lights that moved back and forth just looked like a decoration rather than an indication of intent on the vehicle's part."

\section{Evaluation of Hypotheses}

From these data, we are able to determine the validity of our hypotheses as follows:

H1: Red will be unsuitable as a color to convey a yielding message from an AV's eHMI. On the other hand, green will be intuitive to communicate an AV's yielding intention. The intuitiveness of cyan will fall somewhere in the middle.

Quantitative results show a clear impact of colors on the intuitiveness of an eHMI to communicate a yielding message. Green scored highly, and red scored poorly in intuitiveness, while cyan came in the middle. This was corroborated by the qualitative data - for a vast majority of participants, green corresponded to 'go' and red corresponded to 'stop'. As cyan did not have any innate associations for a message, it was regarded as a neutral color. This leads us to accept $\mathrm{H} 1$.

H2: Wiping or sweeping animation patterns will be more intuitive for an yielding message than a uniformly animating pattern.

As observed from Figure 8, and both the quantitative and qualitative results discussed earlier, uniform animation patterns such as (Flash or Pulse) scored higher in intuitiveness than any of the sweeping patterns in a statistically significant way. Very few participants ascribed the sweeping patterns to a gesture (we revisit this in the Discussion section), and most people found them to be confusing. This leads us to reject $\mathrm{H} 2$.

\section{DISCUSSION}

In this paper, we reported our findings from a survey study how color and animation patterns on a light-band eHMI drive its intuitiveness to communicate a yielding message from an AV. We reflect upon our findings from the quantitative and qualitative results for each independent variable we tested.

\section{Implications of eHMI colors}

The results showed a strong preference for green and a disfavor for red to communicate a yielding message. This was corroborated in the qualitative feedback when a vast majority of the participants noted an innate association of green with an invitation to the pedestrian to cross, and red with a warning or advice for the pedestrian to stop. Some participants commented quite strongly regarding how red is a "terrible color to use" (A43) and green was "perfect" (I11) for communicating yielding. However, some participants did not know what perspective to take while interpreting red or green, while some others confidently ascribed completely reverse intentions to these colors. Several people equated a red light to a brake light in the front of the vehicle and reasoned that it indicated that the car is coming to a stop. On the other hand, some people concluded that a green light indicates the car's intention to move. Despite the imbalance in the associations and the strong overall association with green as a yielding message, this shows that the understanding of red and green, especially on moving AVs, is not universal, and has room for strong confusion. Thus, although we empirically accepted our hypothesis that green may be intuitive as a color to communicate 'yielding' intention, this may not be a good universal solution. This points towards the recommendation that apart from legal and regulatory constraints, red and green may not be suitable for use in eHMI from a user-centered design perspective. Prior research [34] showed positive pedestrian reactions to a green front brake light on a car. However, our research suggests that such implementations are not advisable.

Cyan was regarded as a neutral color, and people were ambivalent about its use. Some observed that cyan was reasonably close to green to be 'passable' as a yielding signal, although not quite as well as green. Others remarked that cyan was neutral and did not tell them anything. Yet others noted that in the absence of cyan from current traffic use, they did not know what to make of the cyan color and it confused them. The lack of a strong association with any contextual meaning allows pedestrians the possibility to learn and assign a new meaning to it without leading to misunderstandings. This therefore leads to a recommendation that cyan may be well-suited for communicating a yielding message in eHMIs.

When participants evaluated different colors of an eHMI for the same animation, they rated green as higher and red as lower in intuitiveness than when they evaluated different animations for the same color (see Figures 5 and 7). Participants' idea of intuitiveness varied more widely between the colors when different colors were presented next to each other than when they saw each color in isolation among different animation patterns for the same color. This effect did not hold for the animation and there was no significant interaction effect. Participants were not consistent in their evaluations of colors and patterns 
across the Measure. However, this also shows that the effect of Color was stronger than the effect of Animation patterns.

The qualitative feedback in the survey reveals that six participants (I45, I68, I139, I148, A57, A103) spontaneously offered suggestions that they would like to see a different color than the green, cyan, and red used in the study; they would like to see yellow or amber as the color to indicate yielding. The association of yellow with a yielding intention is unclear. We know that red and green are the two extremes of the message of a traffic light corresponding to 'stop' and 'go'. On the other hand, the yellow or amber color is traditionally in the middle, and is associated with changing signals which may be regarded as 'standby'. It is possible that the role of yellow in the traffic light was subconsciously extended in the context of a moving vehicle - a vehicle showing yellow or amber light could potentially indicate that it is on standby or it is waiting (i.e. yielding). Yellow as an eHMI color has been explored in the past $[5,22]$. However, its efficacy is unclear, and should be tested in future research. Additionally, yellow and amber lights are already in use on utility, construction, and some emergency vehicles depending on the country, and therefore may be unsuitable for use in eHMI [37].

\section{Impact of animation patterns on eHMI}

Although the main effects for animation patterns showed a statistically significant effect on the intuitiveness of the eHMI, the the effect size was not large. In line with this, the qualitative results showed that animation patterns were regarded as less important than colors. Many participants mentioned that the sweeping patterns caused confusion. The flashing pattern had a similarity with the blinking of hazard lights, and possibly was easy to identify with a 'waiting' message. The pulsing lights were not too different from the flashing pattern, and might have therefore had a similar effect. The sweeping light segments, on the other hand, were associated more with turn signals (similar to the sequential turn signals of some recent cars).

Our hypothesis was that the sweeping lights might map to a waving hand gesture - as if the car is asking the pedestrian to move. Although some feedback was in line with this hypothesis, the majority of the participants did not feel this way. Even within the pool of participants who thought that sweeping animations might relate to gestures, only a small fraction noted that the movement of the light segments mimicked a hand wave. Examples of other interpretations include: a) the InSweep pattern indicated that the car was bowing and asking the pedestrian to go (I107), b) the (OutSweep) indicated the car was "opening a path" and inviting the pedestrian to cross (A167), c) sideways sweeping motion mirrors the motion direction of a pedestrian crossing in front of the car, implying an invitation to cross (I166, A18, A81, A94). From these varying remarks, it appears that sweeping animations do not have a clear and identifiable message. Hensch et al. [24, 25] suggested that eHMI animation is rather unintuitive without prior information. In our study, we extend this knowledge by showing that there does exist a slight user preference for uniform light patterns as opposed to sweeping lights. This is in line with the findings from [14].
These results suggest that more uniform animation patterns could be suitable for showing yielding intention. Laterally animating lights may cause confusion, and may not be ideally suited for communicating intent to yield.

In order to adhere to a symmetry of design, the sweeping movements were all designed to fire in alignment with the center of the car - the light segments began or ended from one end of the bumper to the middle of the bumper. This may have magnified the tendency of participants to liken the sweeping patterns to turn signals. Four participants (A43, A61, A81, A177) also suggested that the ideal pattern would be the one where the light segment alternately moves all the way from one end of the bumper to the other and then returns back. This may be an interesting research question for future work.

\section{Effect of Message}

The kind of Message: Intention communication ["I am yielding"] or Instruction [ "Please cross"] did not have a significant main effect, although there was a significant interaction between Color and the Message. Figure 9 shows that the intuitiveness score for Green in Instruction was higher than the score for Green in the Intention communication condition. Correspondingly, the intuitiveness score of red was lower in the Instruction condition than the Intention Communication condition. This indicates that the suggestive powers of these two colors - green as an invitation to cross, and red as a warning to not cross - are strong. While participants generally ascribed the same meaning in principle to each color in both the conditions, it shows that red and green also have a tendency to be associated more strongly with an instruction from the vehicle, as opposed to a simple announcement of its intention. Given that communication via instruction is not recommended [27], this is yet another reason to discourage the use of red and green in AV eHMIs.

\section{CONCLUSION}

This study utilized online crowdsourcing to explore the role of different colors and animation patterns when used in a light-band external human-machine interface (eHMI) to communicate a yielding message of an automated vehicle. The results suggest that while red and green have immediate associations with 'stop' and 'go', the meaning of these messages is not always clear and may lead to confusion. Cyan, on the other hand, is largely considered a neutral color and may thus be more suitable for communicating a yielding message. Yet another conclusion is that animation patterns do not have as much impact as colors in this communication, although uniform animations may be more favorable than laterally sweeping ones. Given the fact that the role of color and animation for the design of eHMI is currently rather unexplored, our empirical insights might be crucial for the development and standardization of eHMI design in the future.

\section{ACKNOWLEDGMENTS}

This research is supported by the Dutch Domain Applied and Engineering Sciences, which is part of the Netherlands Organization for Scientific Research (NWO), and which is partly funded by the Ministry of Economic Affairs (project number 14896). 


\section{REFERENCES}

[1] Claudia Ackermann, Matthias Beggiato, Sarah Schubert, and Josef F Krems. 2019. An experimental study to investigate design and assessment criteria: What is important for communication between pedestrians and automated vehicles? Applied Ergonomics 75 (2019), 272-282. DOI :

http://dx.doi.org/10.1016/j . apergo.2018.11.002

[2] Jonas Andersson, Azra Habibovic, Maria Klingegård, Cristofer Englund, and Victor Malmsten-Lundgren. 2017. Hello Human, can you read my mind? ERCIM News (109) (2017), 36-37. http:

//urn.kb.se/resolve?urn=urn:nbn: se: ri : diva -29618

[3] Matthias Beggiato, Claudia Witzlack, and Josef F Krems. 2017a. Gap acceptance and time-to-arrival estimates as basis for informal communication between pedestrians and vehicles. In AutomotiveUI 2017 - 9th International ACM Conference on Automotive User Interfaces and Interactive Vehicular Applications, Proceedings. Oldenburg, 50-57. DOI: http://dx.doi .org/10.1145/3122986.3122995

[4] Matthias Beggiato, Claudia Witzlack, Sabine Springer, and Josef Krems. 2017b. The Right Moment for Braking as Informal Communication Signal Between Automated Vehicles and Pedestrians in Crossing Situations. In AHFE 2017. Advances in Intelligent Systems and Computing, vol 597, Vol. 1. 1072 - 1081. DOI : http://dx.doi.org/10.1007/978-3-319-60441-1

[5] Ola Benderius, Christian Berger, and Victor Malmsten Lundgren. 2018. The Best Rated Human-Machine Interface Design for Autonomous Vehicles in the 2016 Grand Cooperative Driving Challenge. IEEE Transactions on Intelligent Transportation Systems 19, 4 (2018), 1302-1307. DOI : http://dx.doi.org/10.1109/TITS.2017.2749970

[6] BMW AG. 2016. BMW Vision Next 100. (2016). https://www. bmwgroup.com/en/next100/brandvisions.html

[7] Michael Buhrmester, Tracy Kwang, and Samuel D. Gosling. 2011. Amazon's mechanical Turk: A new source of inexpensive, yet high-quality, data? Perspectives on Psychological Science 6, 1 (jan 2011), 3-5. DOI : http://dx.doi .org/10.1177/1745691610393980

[8] Daimler AG. 2015. The Mercedes-Benz F 015 Luxury in Motion. (2015).

https://www .mercedes-benz.com/en/mercedes-benz/ innovation/research-vehicle-f-015-luxury-in-motion/

[9] Koen de Clercq, Andre Dietrich, Juan Pablo Núñez Velasco, Joost de Winter, and Riender Happee. 2019. External Human-Machine Interfaces on Automated Vehicles: Effects on Pedestrian Crossing Decisions. Human Factors (2019). DOI : http://dx.doi.org/10.1177/0018720819836343

[10] J. C. F. De Winter and D. Dodou. 2010. FivePoint Likert Items: $t$ test versus MannWhitneyWilcoxon. Practical
Assessment, Research \& Evaluation 15, 11 (2010), 1-16. https://pareonline.net/pdf/v15n11.pdf

[11] Shuchisnigdha Deb, Lesley Strawderman, Daniel W. Carruth, Janice DuBien, Brian Smith, and Teena M. Garrison. 2017. Development and validation of a questionnaire to assess pedestrian receptivity toward fully autonomous vehicles. Transportation Research Part C: Emerging Technologies 84 (nov 2017), 178-195. DOI : http://dx.doi.org/10.1016/j.trc.2017.08.029

[12] Debargha Dey, Marieke Martens, Chao Wang, Felix Ros, and Jacques Terken. 2018. Interface Concepts for Intent Communication from Autonomous Vehicles to Vulnerable Road Users. In Adjunct Proceedings of the 10th International ACM Conference on Automotive User Interfaces and Interactive Vehicular Applications (AutomotiveUI '18). Toronto, Canada, 82-86. DOI : http://dx.doi .org/10.1145/3239092 . 3265946

[13] Djellel Difallah, Elena Filatova, and Panos Ipeirotis. 2018. Demographics and dynamics of Mechanical Turk workers. In WSDM 2018 - Proceedings of the 11th ACM International Conference on Web Search and Data Mining, Vol. 2018-Febua. 135-143. DOI:

http://dx.doi.org/10.1145/3159652 . 3159661

[14] Stefanie M. Faas and Martin Baumann. 2019. Yielding Light Signal Evaluation for Self-driving Vehicle and Pedestrian Interaction. In In: Ahram T., Karwowski W., Pickl S., Taiar R. (eds) Human Systems Engineering and Design II. IHSED 2019. Advances in Intelligent Systems and Computing, Vol. 1026. Springer International Publishing, 189-194. DOI :

http://dx.doi.org/10.1007/978-3-030-27928-8_29

[15] Berthold Färber. 2016. Communication and communication problems between autonomous vehicles and human drivers.. In Autonomous Driving. Springer, Berlin, 125-144.

[16] Andy Field. 2018a. GLM 4: Repeated-Measures Designs. In Discovering Statistics Using IBM SPSS 5th Edition. SAGE Publications, 649-702.

[17] Andy Field. 2018b. GLM 5: Mixed Designs. In Discovering Statistics Using IBM SPSS 5th Edition. SAGE Publications, 703-734.

[18] Ford Motor Corporation. 2017. Ford, Virginia Tech Go Undercover to Develop Signals That Enable Autonomous Vehicles to Communicate with People. (2017). https://media.ford.com/content/fordmedia/fna/ us/en/news/2017/09/13/

ford-virginia-tech-autonomous-vehicle-human-testing . html

[19] Nicolas Guéguen and Céline Jacob. 2002. Direct Look Versus Evasive Glance and Compliance With a Request. The Journal of Social Psychology 142, 3 (2002), 393-396. DOI :

http://dx.doi.org/10.1080/00224540209603907 
[20] Nicolas Guéguen, Sébastien Meineri, and Chloé Eyssartier. 2015. A pedestrian's stare and drivers' stopping behavior: A field experiment at the pedestrian crossing. Safety Science 75 (2015), 87-89. DOI : http://dx.doi.org/10.1016/j.ssci.2015.01.018

[21] Azra Habibovic, Jonas Andersson, Victor Malmsten Lundgren, Maria Klingegård, Cristofer Englund, and Sofia Larsson. 2018a. External Vehicle Interfaces for Communication with Other Road Users? Road Vehicles Automation 5, Lecture Notes in Mobility (2018), 91-102. DOI : http://dx. doi.org/10.1007/978-3-319-60934-8

[22] Azra Habibovic, Victor Malmsten Lundgren, Jonas Andersson, Maria Klingegård, Tobias Lagström, Anna Sirkka, Johan Fagerlönn, Claes Edgren, Rikard Fredriksson, Stas Krupenia, Dennis Saluäär, and Pontus Larsson. 2018b. Communicating Intent of Automated Vehicles to Pedestrians. Frontiers in Psychology 9, August (2018). DOI :

http://dx.doi.org/10.3389/fpsyg.2018.01336

[23] Michael Hamm, Wolfgang Huhn, and Johannes Reschke. 2018. Ideas for Next Lighting Generations in Digitalization and Autonomous Driving - Technocal Paper 2018-01-1038. SAE Technical Papers 2018-April (2018), 1-8. DOI:

http://dx.doi.org/10.4271/2018-01-1038

[24] A Hensch, I Neumann, M Beggiato, J Halama, and J F Krems. 2019a. Steady, Flashing, Sweeping - An exploratory evaluation of light signals as an eHMI in automated driving. (2019). https://www.hfes-europe. org/wp-content/uploads/2019/10/Hensch2019poster.pdf

[25] Ann-Christin Hensch, Isabel Neumann, Matthias Beggiato, Josephine Halama, and Josef F. Krems. 2019b. How Should Automated Vehicles Communicate? âĂŞ Effects of a Light-Based Communication Approach in a Wizard-of-Oz Study. In AHFE 2019. Advances in Intelligent Systems and Computing, vol 964, Vol. 964. Springer International Publishing, 79-91. DOI : http://dx.doi.org/10.1007/978-3-030-20503-4

[26] Kai Holländer, Ashley Colley, Florian Alt, Christian Mai, and Bastian Pfleging. 2019. Investigating the Influence of External Car Displays on Pedestrians ' Crossing Behavior in Virtual Reality. In International Conference on Human-Computer Interaction with Mobile Devices and Services (MobileHCI '19).

[27] International Organization for Standardization (ISO)/TR 23049. 2018. TECHNICAL REPORT ISO: Road Vehicles - Ergonomic aspects of external visual communication from automated vehicles to other road users. Technical Report. https:

//www.iso.org/obp/ui/\#iso:std:iso:tr:23049:ed-1:v1:en

[28] Tobias Lagström and Victor Malmsten Lundgren. 2015. AVIP - Autonomous vehicles' interaction with pedestrians: An investigation of pedestrian-driver communication and development of a vehicle external interface. (2015). http://publications.lib.chalmers.se/ records/fulltext/238401/238401.pdf

[29] Yee Mun Lee, Ruth Madigan, Jorge Garcia, Andrew Tomlinson, Albert Solernou, Richard Romano, Gustav Markkula, Natasha Merat, and Jim Uttley. 2019. Understanding the Messages Conveyed by Automated Vehicles. In 11th International Conference on Automotive User Interfaces and Interactive Vehicular Applications (AutomotiveUI '19). 134-143. DOI : http://dx.doi.org/10.1145/3342197.3344546

[30] Kelly Lin. 2018. 2019 Toyota Avalon Has Sequential Turn Signals. (2018). https://www.motortrend.com/news/ 2019-toyota-avalon-sequential-turn-signals/

[31] Constantin Mircioiu and Jeffrey Atkinson. 2017. A Comparison of Parametric and Non-Parametric Methods Applied to a Likert Scale. Pharmacy 5, 4 (may 2017), 26. DOI: http://dx.doi.org/10.3390/pharmacy 5020026

[32] Jacqueline Murray. 2013. Likert Data: What to Use, Parametric or Non-Parametric? International Journal of Business and Social Science 4, 11 (2013).

[33] Nissan Motor Corporation. 2015. Nissan IDS Concept: Nissan's vision for the future of EVs and autonomous driving. (2015). https://europe .nissannews. com/en-GB/ releases/release-139047

[34] Tibor Petzoldt, Katja Schleinitz, and Rainer Banse. 2018. Potential safety effects of a frontal brake light for motor vehicles. In IET Intelligent Transport Systems, Vol. 12. 449-453. DOI :

http://dx.doi.org/10.1049/iet-its.2017.0321

[35] Amir Rasouli, Iuliia Kotseruba, and John K. Tsotsos. 2017. Agreeing to cross: How drivers and pedestrians communicate. In IEEE Intelligent Vehicles Symposium, Proceedings. Redondo Beach, CA, 264-269. DOI : http://dx.doi.org/10.1109/IVS.2017.7995730

[36] Malte Risto, Colleen Emmenegger, Erik Vinkhuyzen, Melissa Cefkin, and Jim Hollan. 2017. Human-Vehicle Interfaces: The Power of Vehicle Movement Gestures in Human Road User Coordination. In Driving Assessment: The Ninth International Driving Symposium on Human Factors in Driver Assessment, Training and Vehicle Design. Manchester Village, Vermont.

[37] SAE Lighting Standard Practices Committee. 2016. Color Specification (J578 Ground Vehicle Standard) SAE Mobilus. Technical Report. SAE. https://saemobilus.sae.org/content/J578

[38] SAE Standards Works. 2018. J3134 Automated Driving System (ADS) Lamps Task Force. (2018). https: //www . sae.org/works/committeeHome. do?comtID=TEVLCS5Z

[39] S Schmidt and B Färber. 2009. Pedestrians at the kerb Recognising the action intentions of humans. Transportation Research Part F: Traffic Psychology and Behaviour 12, 4 (2009), 300-310. DOI:

http://dx.doi.org/10.1016/j.trf. 2009.02.003 
[40] Friederike Schneemann and Irene Gohl. 2016.

Analyzing driver-pedestrian interaction at crosswalks: A contribution to autonomous driving in urban environments. In IEEE Intelligent Vehicles Symposium, Proceedings. 38-43. DOI :

http://dx.doi.org/10.1109/IVS.2016.7535361

[41] Semcon. 2016. The Smiling Car. (2016). http://semcon.com/smilingcar/

[42] Lenja Sorokin, Nina Kauffmann, and Ronee Chadowitz. 2019. A change of perspective: Designing the automated vehicle as a new social actor in a public space. In CHI'19 Extended AbstractsCHI Conference on Human Factors in Computing Systems Extended Abstracts (CHI'19 Extended Abstracts). DOI : http://dx.doi.org/10.1145/3290607.3299044

[43] Matús Šucha, Daniel Dostal, and Ralf Risser. 2017. Pedestrian-driver communication and decision strategies at marked crossings. Accident Analysis and Prevention 102 (2017), 41-50. DOI :

http://dx.doi .org/10.1016/j . aap. 2017.02.018

[44] Helmut Tiesler-Wittig. 2019. Functional Application, Regulatory Requirements and Their Future Opportunities for Lighting of Automated Driving Systems. Technical Report. DOI :

http://dx.doi.org/10.4271/2019-01-0848
[45] UNECE (United Nations Economic Commission for Europe). 2011. UNECE R65 Regulation - Uniform provisions concerning the approval of special warning lamps for power-driven vehicles and their trailers. Technical Report December. UNECE. 1-29 pages.

[46] UNECE (United Nations Economic Commission for Europe). 2018. Autonomous Vehicle Signalling Requirements (AVSR) Taskforce. (2018). https://wiki. unece. org/pages/viewpage . action?pageId=73925596

[47] Viknesh Vijayenthiran. 2013. Audi Shows Sequential Turn Signal Of 2015 A8. (2013). https://www .motorauthority. com/news/1086249

[48] Erik Vinkhuyzen and Melissa Cefkin. 2016. Developing Socially Acceptable Autonomous Vehicles. Proceedings of the Ethnographic Praxis in Industry Conference (EPIC) (2016), 423-435.

[49] Annette Werner. 2018. New Colours for Autonomous Driving: An Evaluation of Chromaticities for the External Lighting Equipment of Autonomous Vehicles. ColourTurn 10, 3 (2018), 183-193. DOI : http://dx.doi.org/10.25538/tct.v0i1. 692

[50] Christopher D Wickens, John Lee, Viii Uu, and Sallie Gordon Becker. 2004. An Introduction to Human Factors Engineering Second Edition (2 ed.). Pearson. 